\title{
УДК $533.73+57.037$
}

\section{Влияние теплопроводности кожных покровов на тепловое состояние человека при общей газовой криотерапии}

\author{
Канд. техн. наук Левин М.Л. marklvn@ belhard.com \\ Канд. физ.-мат наук Маханёк А.А. amakh@tut.by \\ Институт тепло- и массообмена им. А.В. Лькова \\ Наџиональной академии наук Беларуси \\ 220072, Беларусь, Минск, П. Бровки ул., 15
}

\begin{abstract}
Рассмотрено влияние теплопроводности кожи пациента на его тепловое состояние во время и после окончания процедуры общей газовой криотерапии (ОГКТ). Теплоперенос в организме человека описывался в рамках 19-компартментной модели Гордона для стандартного человека, модифицированной с целью учета индивидуальных антропометрических особенностей конкретного пациента (рост, масса, относительная доля жсировой и мышечной ткани и т.д.). Вычисление коэффициентов теплоотдачи от цилиндрических компартментов осуществлялось согласно справочным формулам. Представлены результаты экспериментальных исследований различий по температуре кожных покровов у молодых (21 год) и высоковозрастных (69 лет) спортсменов после процедуры ОГКТ в сравнении с результатами вычислительного эксперимента. Показано, что измеренные и вычисленные температуры кожи двух возрастных групп людей различаются менее чем на 0,2 ${ }^{\circ}$, , а различие между температурой кожи жсивота у молодых и возрастных пациентов составляет $4{ }^{\circ} \mathrm{C}$ при температуре газового хладагента (воздуха) минус $110{ }^{\circ}$ С и продолюсительности ОГКТ 3 мин. Сделан вывод, что в широком диапазоне возможсных относительных возрастных изменений интенсивности основного обмена, толицны подкожной жировой клетчатки и теплопроводности кожи именно изменение теплопроводности дает основной вклад в различие температур поверхности кожи молодых и пожилых пациентов. Ключевые слова: общая газовая криотерапия, тепловая задача, теплоперенос, теплопроводность кожи, возраст пациента.
\end{abstract}

DOI:10.17586/2310-1148-2017-10-1-8-14

\section{The effect of age of the patient at whole-body gas cryotherapy}

\author{
Ph.D. Levin M.L.marklvn@belhard.com \\ Ph.D. Makhaniok A.A. amakh@tut.by \\ A.V. Luikov Heat and Mass Transfer Institute \\ National academy of sciences of Belarus \\ 220072, Belarus, Minsk, P. Brovki St., 15
}

The influence of the skin thermal conductivity of the patient on his heat condition during and after procedure of whole body gas cryotherapy (WBGC) has been examined. Heat transfer in human body was characterized within the bounds of 19-compartment Gordon model for a "standard" human being which was modified purposely with due regard for individual anthropometric data as stature, body weight, relative adipose and muscular tissue and others. Computation of heat-transfer coefficients of cylindrical compartments was realized according to inquiry formulas. Results of experimental investigations of difference of temperature of cutaneous coverings of young (21 years old) and high aged (69 years old) sportsmen after WBGC procedure in comparison with the results of computing experiment are presented. It has been revealed that measured and calculated skin temperatures for two age groups differ less than $0,2^{\circ} \mathrm{C}$ and difference between skin temperature of abdomen compartment of young and high aged patients was $4{ }^{\circ} \mathrm{C}$ at the temperature of gas cooling medium (air) minus $110{ }^{\circ} \mathrm{C}$ and 3 minutes of WBGC time exposure. We concluded that in wide-ranging possible relative age-related changes of basal metabolic rate, 
thickness of hypoderm and thermal conduction of skin, exactly thermal conduction changing brings in capital contribution of difference of temperature of cutaneous covering of young and high aged patients. Keywords: whole-body gas cryothepy, heat problem, heat transfer, skin heat conductivity, age of patient.

\section{Введение}

Общая газовая криотерапия (ОГКТ) применяется для оздоровления, лечения и повышения работоспособности пациентов разных возрастов. С возрастом у человека происходит ряд изменений, потенциально способных повлиять на тепловое состояние пациента во время ОГКТ и эффект применения данной процедуры. Так, влияние возраста может проявиться в силу зависимости от него уровня основного обмена, а также имеющейся статистической связи между возрастом и количеством жировой ткани у человека [1]. Рост, масса и полнота человека обуславливают площадь теплообменной поверхности, что также немаловажно для ОГКТ. В настоящее время существующие протоколы применения ОГКТ возрастной фактор не учитывают. Целью данного исследования являлась оценка влияния данного фактора на температуру кожных покровов пациента при ОГКТ.

\section{Постановка тепловой задачи}

Тело человека представляется в виде множества цилиндров, состоящих из четырех слоев биоткани: ядра, мышечного слоя, слоя подкожной жировой клетчатки и кожи (в компартментах головы вместо мышечного слоя задается слой кости). Ядро составляют мягкие ткани в компартментах «голова», «лоб», «лицо», «грудная клетка» и «живот», или кость в случае конечностей (компартменты «плечо», «предплечье», «кисть», «бедро», «голень», «ступня»). Характерные размеры компартментов и их слоев (субкомпартментов) для «стандартного человека» определены в работах [2, 3] и обеспечивают реалистичное описание массы, объема, теплосодержания и теплообмена такого человека в рамках модели с сосредоточенными параметрами [4, 5]. В основу антропометрической модели положена модифицированная нами модель Гордона [6]. Отличия связаны с детализацией некоторых компартментов, а также в реализации пересчета толщин слоев при отклонении массы и роста пациента от значений, характерных для «стандартного» человека:

- длина торса, бедра и голени вычисляется пропорционально росту человека с коэффициентам пропорциональности из таблицы 1 в [5];

- пропорции длин компартментов грудная клетка и живот такие же, как и в модели Гордона;

- радиусы слоев для предплечья и локтевой части руки, а также бедра и голени корректируются так, чтобы их объемы соотносились как в [6];

- при варьировании массы пропорционально изменяются массы, объемы и внешние радиусы всех слоев, исключая голову;

- толщина кожного покрова для разных участков, такая же, как в [6] и не зависит от возраста и комплекции человека.

Постановка тепловой задачи для $k$-го слоя $j$-го компартмента имеет вид:

$$
\begin{gathered}
\left(\rho C_{p}\right)_{j k} \frac{\partial T_{j k}}{\partial t}=\nabla\left(\lambda_{j k} \nabla T_{j k}\right)+Q_{j k} \\
\left.T_{j k}\right|_{\Gamma_{j k}}=\left.T_{j, k-1}\right|_{\Gamma_{j k}} ;\left.\quad \lambda_{j k} \nabla T_{j k}\right|_{\Gamma_{j k}}=\left.\lambda_{j, k-1} \nabla T_{j, k-1}\right|_{\Gamma_{j k}}, \quad k=1,2,3 \\
-\left.\lambda_{j k} \nabla T_{j k}\right|_{\Gamma_{j 4}}=\alpha_{j}\left(\left.T_{j k}\right|_{\Gamma_{j 4}}-T_{e n v}\right) \\
T_{j k}(t=0)=T_{j k}^{0}
\end{gathered}
$$

Слагаемое $Q_{j k}$ в уравнении энергии (1) учитывает мощность внутренних источников и стоков тепла, в частности, внутритканевый конвективный (перфузионный) теплообмен $Q_{c o n v, j k}=C_{b} M_{j k} W_{j k}\left(T_{b}-T_{j k}\right)$ [7], интенсивность метаболической теплопродукции $Q_{\text {met, } j k}=Q_{\text {met, }, \text { as }} p^{\left(T_{j k}-T_{\text {bas }, j k}\right) / T_{\text {ref }}}$, где $p=2, T_{r e f}=10^{\circ} \mathrm{C}[6]$ (для головного мозга $p=3$ [8]). Другие детали постановки тепловой задачи (в том числе выражения, описывающие $Q_{j k}$ в разных компартментах), а также многочисленные геометрические и теплофизические параметры и источники, из которых они были взяты, приведены в $[4,9]$. Отметим выражения, явным образом учитывающие влияние 
возраста пациента на соответствующий параметр модели. Так, зависимость массовой доли жира от возраста описывается формулой [10]

$$
f=0,011,2 B M I+0,23 Y-5,4(2 g+1),
$$

где, $B M I=M / H^{2}$ - приведенный индекс массы (масса $M$ тела выражена в кг, а рост $H-$ в см); $Y$ - возраст человека, лет; $g=1$ в случае мужчины и $g=0$ для женщины.

При моделировании зависимости удельного метаболического теплообразования от возраста (учитываемого параметром $Q_{j k}$ ) исходим из соотношения

$$
Q_{m e t, b a s}=Q_{m, b a s} Q_{m e t, b a s}^{*}(Y) / Q_{m e t, b a s}^{*}\left(Y_{0}\right),
$$

где $Y_{0}$ - характерный возраст, принятый нами равным 30 лет, параметр $Q_{m, b a s}$ зависит от типа биоткани, а $Q_{\text {met }, \text { bas }}^{*}(Y)=84.78-1.99 Y+0.0405 Y^{2}-3.0071 \cdot 10^{-3} Y^{3}$ Вт $[2,11]$.

Изменение кровотока в ходе нагрева описывается терморегуляционной моделью Столвика [12]. Согласно этой модели расширение и сужение кровеносных сосудов в слоях кожи зависят от знака средней температуры кожи всего тела, гипоталамической температуры головы (центральной части) и локальной температуры каждого участка кожи. Учет возраста в базисных кровотоках для каждого субкомпартмента осуществляется подобно [5]:

$$
W_{b, j k}=W_{b, j k}^{\prime} \frac{C O_{b a s}(Y)}{C O_{b a s}\left(Y_{0}\right)},
$$

где $\quad W_{b}^{\prime}, j k-$ значения перфузии в каждом субкомпартменте, относящиеся к «стандартному» человеку; $C O_{\text {bas }}$ величина сердечного выброса в термонейтральных условиях. Зависимость величины сердечного выброса от возраста описывается выражением [2]

$$
C O_{b a s}=\left(240,691-2,716 Y+0,0157 Y^{2}\right) B S A,
$$

в котором $B S A$ - площадь поверхности кожи.

Учет изменения интенсивности кровотока в биоткани при ее нагреве/охлаждении осуществляется в параметре $W_{b, j k}^{\prime}$ согласно модели Столвика-Харди [12]. Теплофизические свойства биоткани (плотность $\rho$, теплоемкость $C_{p}$, теплопроводность $\lambda$ ) в пределах одного слоя полагаются однородными и независящими от температуры.

В рамках нашего варианта антропометрической модели Гордона предусмотрено переопределение геометрических характеристик компартментов и отдельных слоев на произвольные значения роста, массы человека и толщины подкожной жировой прослойки. Массы слоев всех компартментов, кроме головы и шеи, изменяются пропорционально различию заданной массы и роста относительно массы и роста так называемого "стандартного" человека. При увеличении внешних радиусов слоев мышечной биоткани уменьшается толщина подкожной жировой прослойки. Аналогично, пересчет внешних радиусов слоев мышц, жира и кожи при изменении массы подкожного жира (заданной в относительных единицах) производится за счет соответствующего изменения массы мышц. Массовая доля жира для не спортсменов вычисляется по формуле (5). Для спортсменов она определяется исходя из заданной массовой доли мышечной ткани, массы тела и роста.

Выражения (1) - (4) описывают непрерывность поля температур и тепловых потоков на границе слоев биоткани (ядро-мышцы, мышцы-жировой слой, жировой слой-кожа), теплообмен кожных покровов с окружающим их газовым хладагентом и начальное условие. Определяется начальное распределение температур из решения аналогичной задачи для нескольких этапов, в том числе определение установочных (базисных) температур, необходимых для применения терморегуляционной модели Столвика [12, 13]. Эти температуры характерны для конкретного человека с его специфическими антропометрическими и возрастными данными. Решается стационарная тепловая задача

1) с начальной температурой тела, равной $37^{\circ} \mathrm{C}$;

2) пребывание обнаженного пациента при комнатной температуре $22^{\circ} \mathrm{C}$ в течение 15 мин;

3) адаптация пациента к низкой температуре в предкамере криосауны при минус $60{ }^{\circ} \mathrm{C}$ на протяжении 30 сек (третий этап); 
4) нахождение пациента в основной процедурной камере при минус $110{ }^{\circ} \mathrm{C}$ в течение 3 мин (режим проведения ОГКТ в криокамере Cryospace), или другие сочетания температуры газового хладагента $T_{e}$ и продолжительности холодовой процедуры.

Коэффициенты теплоотдачи вычислялись по справочным данным [14] для условий естественной конвекции. Предварительно производилась оценка величин чисел Рэлея $R a$ и в случае, когда $R a>10^{9}$ числа Нуссельта $N u$ (и коэффициенты теплоотдачи $\alpha$ ) определялись для турбулентного режима течения газового хладагента. Эффективный коэффициент теплоотдачи, входящий в граничное условие (3), учитывает как конвективную, так и радиационную составляющую. Сформулированная выше тепловая задача позволяет получить распределение температур, как в теле человека, так и по поверхности его отдельных частей тела с учетом основных механизмов терморегуляции (дрожания, тепла метаболизма и др.).

Влияние возраста в нашей физико-математической модели ОГКТ учитывается три фактора:

- влияние возраста на интенсивность основного обмена и тканевого кровотока согласно формулам (6) - (8),

- увеличение с возрастом массовой доли подкожной жировой клетчатки по формуле (5) и снижение с возрастом теплопроводности кожи от 0 до $60 \%$ у семидесятилетних пациентов по сравнению с двадцатилетними согласно линейной модели: $\lambda(Y)=\lambda\left(Y_{0}\right) C_{\lambda}(Y)$, в которой возрастная поправка в теплопроводность кожи

$$
C_{\lambda}(Y)=\frac{50+\varepsilon_{C}(70-Y)}{50+\varepsilon_{C}\left(70-Y_{0}\right)},
$$

а параметр $\varepsilon_{C}=\lambda(20) / \lambda(70)-1$ отражает относительное уменьшение коэффициента теплопроводности кожи у пожилых пациентов (возрастом 70 лет) по сравнению с молодыми (возрастом 20 лет). При моделировании влияния возраста на толщину подкожной жировой клетчатки полагали, что 4\% жира у мужчин и $12 \%$ жира у женщин связано с внутренними органами, т.е. результат, даваемый формулой (5), уменьшали на эти величины.

\section{Результаты и их обсуждение}

Количественное значение параметра $\varepsilon_{C}$ не известно. Для его оценки были использованы собственные экспериментальные данные, полученные на спортсменах высокой квалификации двух возрастных групп со средними возрастами 21 год и 69 лет. Модельные данные по спортсменам (мужчины) приведены в табл. 1. Они получены при задании доли мышечной массы 55 \% для молодых спортсменов и 40 \% - для высоковозрастных спортсменов, что, по нашим оценкам, примерно соответствует результатам, полученным с помощью биоимпедансного анализатора состава тела MC-180 японской фирмы Tanita [15], с учетом некоторого завышения этим прибором доли мышечной массы. Формула (5) при этом не использовалась. Измеренные средние по поверхности кожи компартмента «живот» значения температур у молодых и высоковозрастных спортсменов на момент окончания ОГКТ составляли $18,94^{\circ} \mathrm{C}$ и $14,90^{\circ} \mathrm{C}$, соответственно.

Таблица 1

\section{Расчетные антропометрические характеристики спортсменов}

\begin{tabular}{|l|c|c|}
\hline \multicolumn{1}{|c|}{ Параметр } & $\begin{array}{c}\text { Молодые } \\
(21 \text { год) }\end{array}$ & $\begin{array}{c}\text { Высоковозрастные } \\
\text { (69 лет) }\end{array}$ \\
\hline Рост, см & 185 & 175 \\
\hline Масса тела, кг & 85,7 & 92,3 \\
\hline Площадь поверхности кожи, м² & 2,083 & 2,143 \\
\hline Масса мягких тканей ядра, кг (\% от массы тела) & $22,97(26,8)$ & $23,39(25,3)$ \\
\hline Масса костей, кг (\% от массы тела) & $10,40(12,1)$ & $13,74(14,9)$ \\
\hline Масса мышц, кг (\% от массы тела) & $45,91(53,6)$ & $36,56(39,6)$ \\
\hline Масса подкожной жировой клетчатки, кг (\% от массы тела) & $2,26(2,6)$ & $15,34(15,5)$ \\
\hline Масса кожи, кг (\% от массы тела) & $4,17(4,9)$ & $4,29(4,7)$ \\
\hline
\end{tabular}


Сформулированная выше тепловая задача решалась при варьировании параметра $\varepsilon_{C}$ в диапазоне $0-0,6$. Для обеспечения наилучшего приближения расчетных температур к экспериментальным коэффициент теплоотдачи от поверхности кожи был уменьшен на 10 \% по сравнению с расчетными значениями, получаемыми по справочными данным [14].

Отклонение расчетных температур от измеренных составило примерно $0,2{ }^{\circ} \mathrm{C}$, а отличие в расчетных температурах $4,01{ }^{\circ} \mathrm{C}$ при значении параметра $\varepsilon_{C}$, равном 0,55 . Столь значительное различие в теплопроводностях кожи спортсменов молодого и старшего возраста может быть обусловлено не только возрастным фактором, но и различием в фактической толщине слоя кожи и величине базового кровотока, неоднородностью теплофизических свойств кожи по ее толщине, несовершенством антропометрической и тепловой модели. Также возможно отличие фактических толщин подкожной жировой клетчатки от вычисленных из модельных представлений и нелинейность влияния возраста на теплопроводность кожи. Все это ведет к огрублению нашей оценки относительного изменения эффективной теплопроводности кожи с возрастом. По этой причине моделирование теплового состояния не спортсменов и относительного вклада трех рассматриваемых механизмов возрастного влияния на тепловое состояние пациента после ОГКТ осуществлялось при двух значениях параметра $\varepsilon_{C}: 0,25$ и 0,5. Количественно этот вклад показан на рисунке 1. Методика искусственного вычленения каждого из рассматриваемых механизмов возрастного влияния на тепловое состояние пациента была следующей. Решение тепловой задачи (1) - (4) осуществлялось в четырех вариантах: 1 - с учетом зависимости от возраста метаболизма, кровотока, толщины подкожного жира и теплопроводности кожи, 2 - аналогично 1 , но при постоянной теплопроводности кожи, соответствующей возрасту $Y_{0}=30$ лет, 3 - с учетом зависимости от возраста только толщины подкожного жирового слоя, 4 - аналогично 3, но с учетом также влияния возраста на теплопроводность кожи согласно модели (9) при заданном значении параметра $\varepsilon_{C}$. Эффект (различие в температурах поверхности кожи компартмента «живот» пациентов возрастом 20 и 70 лет), полученный в варианте 3, вычитался из эффектов, получаемых в вариантах 2 и 4, так как невозможно абстрагироваться от антропометрических характеристик, а они обусловлены, в том числе, и возрастом пациентов.

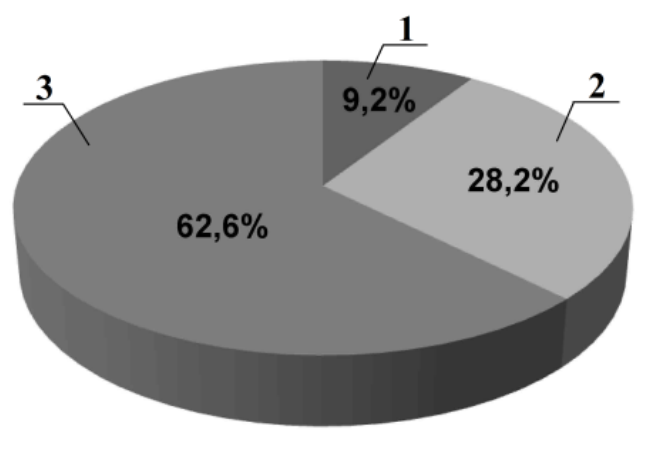

$a$

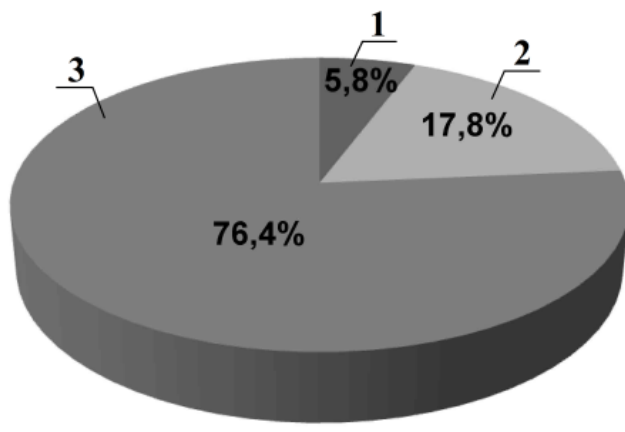

$\sigma$

Рис. 1. Относительный вклад в возрастное различие температуры поверхности кожи не спортсменов с телосложением $180 x 80$ при различии в теплопроводности кожи двадияати и семидесятилетних пациентов 25\% (а) и 50\% (б):

1 - изменение с возрастом только интенсивности основного обмена и кровотока;

2 - возрастное изменение только доли жира соответственно формуле (5);

3 - изменение с возрастом лишь теплопроводности кожи согласно модели (9)

Можно констатировать, что в широком диапазоне возможных относительных возрастных изменений теплопроводности кожи именно этот механизм дает основной вклад в различие температур поверхности кожи молодых и пожилых пациентов. Величина вклада других механизмов влияния возраста на тепловое состояние пациента зависит от величины параметра $\varepsilon_{C}$, но всегда эффект от возрастного изменения толщины подкожной жировой клетчатки значительно сильнее, нежели от снижения с возрастом интенсивности основного обмена.

Режимы проведения ОГКТ на сегодняшний день устанавливаются исходя из опыта разработчиков криотерапевтического оборудования, либо проводящих эту процедуру специалистов-медиков. В зависимости от вида криотерапевтической установки и психофизиологической подготовленности пациента они могут различаться весьма значительно. В любом случае отсутствуют какие либо обобщения этих режимов и рекомендации относительно оптимальных сочетаний продолжительности холодового воздействия и температуры газового хладагента в зависимости от антропометрических характеристик и других индивидуальных особенностей пациентов. Судя по отмеченному выше эффекту существенного влияния возраста человека на температуру поверхности его кожи после ОГКТ целесообразно рассмотрение влияния возрастного фактора на тепловое состояние пациента в широком диапазоне сочетаний основных режимных параметров: 
продолжительности данной процедуры и температуры газового хладагента. Результат такого рассмотрения представлен на рисунке 2 для молодых (20 лет) и пожилых (70 лет) пациентов с телосложением $180 x 80$.

Моделирование выполнено при задании величины параметра $\varepsilon_{C}=0,5$. Отметим линейную зависимость вычисленной температуры поверхности кожи компартмента «живот» от продолжительности процедуры и очень слабую нелинейность зависимости этого параметра от температуры газового хладагента. У пожилых пациентов наблюдается более низкая температура поверхности кожного покрова по сравнению с молодыми. Разница температур поверхности кожи между молодыми и пожилыми пациентами усиливается по мере понижения температуры в криокамере и не зависит от продолжительности холодового воздействия. При рассмотренных режимах для пациента с телосложением 180х80 отрицательные температуры на поверхности кожи живота обнаруживаются лишь у 70-летних пациентов при температуре газового хладагента ниже минус $150{ }^{\circ} \mathrm{C}$ и продолжительности ОГКТ 4 мин. Различия в температурах кожных покровов пациентов сходной комплекции, но разного возраста усиливаются по мере снижения температуры газового хладагента и продолжительности ОГКТ. Из этой закономерности следует, что при формировании протокола проведения ОГКТ для обеспечения безопасности данной процедуры следует оговаривать предельный возраст пациентов, либо ориентироваться на самых старших по возрасту пациентов в заданной возрастной группе. Наиболее рациональным представляется выбор сочетаний продолжительность ОГКТ - температура в криокамере для нескольких возрастных групп, например, для диапазона возрастов 20-30 лет, 30-40 лет и т.д. с ориентацией на верхнюю границу возраста в каждой группе.

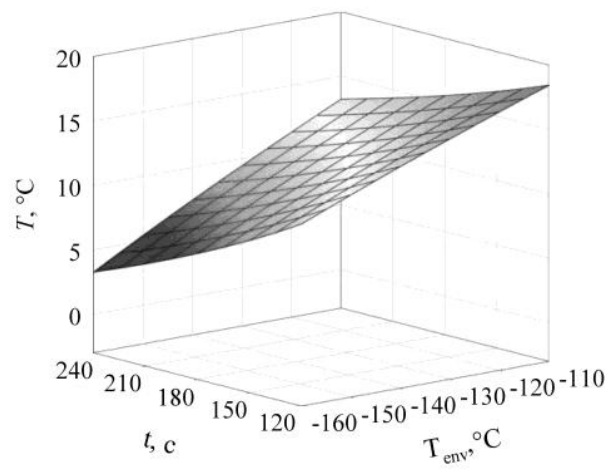

$a$

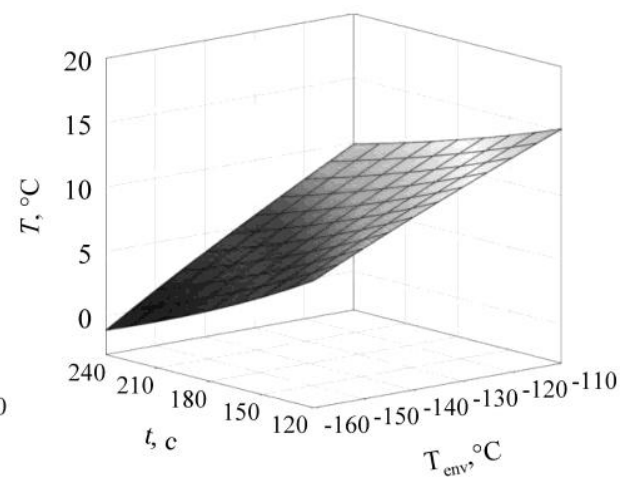

$\sigma$

Рис. 2. Температура поверхности кожи компартмента «живот» пациентов возрастом двадияать (а) и семьдесят (б) лет с телосложением 180x80

при разных сочетаниях продолжительности ОГКТ (t) и температуры газового хладагента ( $\left.T_{\text {епv }}\right)$

Более низкая температура кожных покровов пациентов старшего возраста при одинаковых с более молодыми пациентами сочетаниях роста и массы тела вовсе не означает, что и положительный (лечебный) эффект холодового воздействия на людей старшего возраста будет более выражен. На сегодняшний день физиологические и другие механизмы лечебного воздействия ОГКТ досконально не определены. Они могут быть обусловлены не только низкой температурой кожи, но и огромными потоками теплоты, отводимой от тела пациента в криокамере во время процедуры ОГКТ.

При одинаковых телосложениях более выраженный холодовый стресс (согласно нашей модели ОГКТ) должны испытывать молодые пациенты, так как при одинаковых ростомассовых характеристиках (комплекциях) меньшему возрасту соответствует и меньшая толщина подкожной жировой прослойки, а величина теплового потока через кожу молодых пациентов оказывается выше, чем у более пожилых пациентов. Возрастной фактор, по результатам нашего исследования, оказывает значительное влияние на достигаемую величину охлаждения кожных покровов пациента. Однако этот факт не означает наличие более сильного эффекта от применения ОГКТ у пожилых пациентов по сравнению с молодыми, так как величина теплового потока отводимого от кожи таких пациентов меньше, чем у более молодых. По этой причине является целесообразным проведения анализа эффективности процедуры ОГКТ в сравнении лечебного эффекта в разных возрастных группах. В связи с тем, что более выраженное понижение температуры кожных покровов у пожилых людей сопровождается некоторым уменьшением величины теплопотерь (по сравнению с более молодыми пациентами такой же комплекции), еще большую актуальность приобретает вопрос о механизмах лечебного эффекта. В частности, какой из теплофизических факторов с точки зрения повышения эффективности данной холодовой терапии является значимым: более низкая температура кожи или более высокий тепловой поток. 
Уменьшение теплопроводности кожи с возрастом обусловлено главным образом уменьшением влагосодержания в кожных покровах, которое зависит не только от возраста. Его величина может быть обусловлено уровнем физической нагрузки, если такая нагрузка предшествовала процедуре ОГКТ, а также влиянием психофизических факторов (волнение, переживание, боязнь и т.п.). Второй по влиянию на температуру кожного покрова фактор - толщина слоя подкожной клетчатки. Если толщина кожи в одинаковых местах у разных людей примерно одинакова, то толщина подкожной жировой клетчатки и создаваемого ею теплового сопротивления могут варьировать в очень широких пределах, еще больше усиливая при этом влияние возрастного фактора при уменьшении толщины подкожного жира, или, наоборот, ослабляя эффект возрастного снижения теплопроводности кожи в случае ожирения.

\section{Выводы}

Вычислительный эксперимент показал, что наблюдается значительная зависимость величины изменения температуры поверхности кожи разных компартментов у 70-летних пациентов по сравнению с 20-летними. Обнаруженный эффект качественно и количественно подтвержден экспериментально результатами термографического мониторинга теплового состояния 20-летних и 70-летних спортсменов. При одинаковых телосложениях более выраженный холодовый стресс испытывают более молодые пациенты, так как с уменьшением возраста величина теплового потока отводимого от поверхности кожного покрова пациента возрастает.

\section{Литература (References)}

1. Gehan, E.A. Estimation of Human Body Surface Areas from Height and Weight / E.A. Gehan, S.L. George // Cancer Chemotherapy Reports. 1970. Vol. 54. P. 225-235.

2. Arkin, H. Model of thermoregulation in the human body. Part III: Model behavior and comparison to experimental results of exercising, heat / Arkin H., Shitzer, A. // EEC-150. Depth. of Mechanical Engineering Technion, Haifa, Israel. 1984. P.1-36.

3. Stolwijk, J.A.J. A mathematical model of physiological temperature regulation in man / J.A.J. Stolwijk. NASA CR1855. Yale University school of medicine. Washington, 1971. 77P.

4. Charny, C.K. A whole body thermal model of man during hyperthermia tissues / C.K. Charny, M.J. Hagmann, R.L. Levin // IEEE Trans. on Biomed. Eng. 1987. Vol. BME-34. P. 375-386.

5. Gordon, R.G. A Mathematical model for the human temperature regulatory system - transient cold exposure response / R.G. Gordon, R.B. Roemer, S.M. Horvath // IEEE Trans. on Biomed. Engng. 1976. Vol. BME-23, P. 434-444.

6. Маханёк, А.А. Теплофизические аспекты общей газовой криотерапии / А.А. Маханёк, М.Л. Левин, В.Л. Драгун // Весці НАН Беларусі. Сер. фіз.-тэхн. навук. 2011. № 3. С. 59-71.

7. Pennes H.H. Analysis of tissue and arterial blood temperature in the resting human forearm / H.H. Pennes // J.Appl. Physiol. 1948. Vol. 1. P. 93.

8. Xu X. A mathematical model for human brain cooling during cold-water near-drowning / X. Xu, G. Giesbrecht // J. Appl. Physiol. 1999. Vol. 86. P. 265-272.

9. Левин, М.Л. Влияние некоторых факторов теплообмена при общей криотерапии на температуру кожных покровов / М.Л. Левин, А.А. Маханёк, В.Л. Драгун // II научно-практическая конф. «Криотерапия в России», Санкт-Петербург, 14 мая 2009. С. 80-103.

10. Deurenberg, P. Body mass index as a measure of body fatness: age- and sex-specific prediction formulas / P. Deurenberg, J.A. Weststrate, J.C. Seidell // Br.J. Nutr. 1991. Vol. 65. P. 105-114.

11. Arkin, H. Model of thermoregulation in the human body. Part I: The heat transfer model (The "passive" model) / Arkin H., Shitzer A. // EEC-148. Depth. of Mechanical Engineering Technion, Haifa, Israel. 1984. P. 1-63.

12. Stolwijk J.A.J. Temperature regulation in man - A theoretical study / J.A.J. Stolwijk, J.D. Hardy // Pflugers Arch. 1966. Vol. 291. P. 129-162.

13. Stolwijk, J.A.J. Mathematical models of thermal regulation / J.A.J. Stolwijk. In Thermal characteristics of tumors: applications in detection and treatment. Ed. R.K. Jain, P.M. Gullino. Annals of the New York Academy of Sciences. 1980, Vol. 335, P. 98-106.

14. Тепло- и массообмен. Теплотехнический эксперимент. Справочник. Под ред. В.А.Григорьева и В.М.Зорина. М.:Энергоиздат, 1982. С.177-178.

15. Левин М.Л. Термографический мониторинг при локальной и общей газовой криотерапии / М.Л. Левин, А.А. Маханёк, С.М. Данилова-Третьяк, В.Г. Лещенко // Препринт № 4. Институт тепло- и массообмена. Минск. 2014. 61c. 